\title{
Energy simulations on switchable mirrors - comparisons between three simulation tools
}

\author{
Andreas Jonsson ${ }^{1, *}$, Arne Roos ${ }^{1}$, Yamada Yasusei $^{2}$ \\ ${ }^{1}$ Uppsala University, Uppsala, Sweden \\ ${ }^{2}$ National Institute of Advanced Industrial Science and Technology, Nagoya, Japan \\ *Corresponding author. Tel: +46 184901215, Fax: +46 184713270, E-mail: andreas.jonsson@angstrom.uu.se
}

\begin{abstract}
In a research collaboration between the National Institute of Advanced Industrial Science and Technology (AIST) in Japan and Uppsala University switchable mirrors have been evaluated from an energy perspective using energy simulations for smart windows based on gasochromic switchable mirrors. Optical properties and $\mathrm{U}$ values for the transparent and reflectivestates are used as input for the energy simulations. A test room has been built and is located in Nagoya, Japan. Simulations on energy use for smart windows based on switchable mirrors have been compared between three energy simulation tools. The simulations show good agreement and the simulation results also address the importance of a good control strategy for the smart windows.
\end{abstract}

Keywords: Switchable Mirrors, Smart Windows, Energy Simulations, Gasochromic windows

\section{Introduction}

A step towards a society not based on fossil fuels might be to use more renewable energy. Another step is energy conservation. Smart or switchable mirrors can be one way to conserve energy within buildings. They can reduce cooling needs in warm and/or varying climates since the transmittance of light and hence the solar heat gain factor can be reduced. This reduction can be achieved when people are present to give a comfortable level of daylight and when people are not present adapted to a level which leads to the lowest energy need for the building. The control strategy of such windows is important for their energy performance [1, 2].

In this study the focus has been on smart windows based on gasochromic switchable mirrors based on metal hydrides [3]. The state of the window is changed using a gas mixture with argon and hydrogen to turn the window to a transparent state and using a gas mixture with oxygen to revert the window back to a reflective state. This means that it is necessary to combine a switchable glass in a window of at least two panes and control the window by letting in either gas mixture in between the panes.

\section{Methodology}

\subsection{Energy simulations}

Proper energy simulations on smart windows require that the simulation tool can handle building components with varied properties over time. In this project three simulation tools were used. WinSel is a static window simulation tool developed at Uppsala University, VIP Energy [4] is a commercial dynamic building simulation tool and eQuest [5], which is also a dynamic building simulation tool developed by Lawrence Berkeley National Laboratory. Meteorological input data have been obtained from the software tool Meteonorm [6].

\subsubsection{WinSel}

WinSel is a software tool for evaluating and comparing windows. The software calculates the energy for heating and cooling caused by the windows as a building component. The purpose 
is to be a simple tool for selecting windows. Using the window properties solar gain and $U$ value, different windows can be compared for a building located in a specific climate using just balance temperature and a climate data file as input. The WinSel simulation parameters can be found in table 1. Due to the simplicity of the program, it is suitable as a tool for selecting the right type of window for a certain building. The result achieved from the program is the energy balance for the heating season and the cooling season. The energy balance is calculated per square meter glazing area from the equation:

Energy balance $=$ Solar heat gain - Thermal losses

Table 1. WinSel simulation parameters.

\begin{tabular}{cc}
\hline Parameter & Value \\
\hline Climate (from Meteonorm 5.0): & Nagoya \\
Ground reflection & $20 \%$ \\
Building balance temperature & $12^{\circ} \mathrm{C}$ \\
Allowed temperatures & $20-26^{\circ} \mathrm{C}$ \\
& \\
U value, transparent state & $2,40 \mathrm{~W} / \mathrm{m}^{2} \mathrm{~K}$ \\
g-value, transparent state & $50 \%$ \\
T_vis, transparent state & $35 \%$ \\
& \\
U value, reflective state & $1,67 \mathrm{~W} / \mathrm{m}^{2} \mathrm{~K}$ \\
g-value, reflective state & $6 \%$ \\
T_vis, reflective state & $5 \%$ \\
\end{tabular}

WinSel can simulate smart windows with variable solar gain, g-value, and variable thermal performance, $U$ value. In the software the g-value and the $U$ value can be time dependent and are regulated using different control strategies. Six different control strategies have been developed to exemplify different approaches for controlling smart windows:

EO - "Energy optimization" means that the windows are always kept in the state which is best from an energy perspective. In the simulations the windows are kept in mirror state whenever there is a cooling need and in a transparent state whenever there is a heating need.

DO - "Daylight optimization" means that the windows are in a state which is optimized from a daylight perspective. The perpendicular component of the transmitted direct solar radiation was thus regulated by the switchable mirror in the window to a maximum of $200 \mathrm{~W} / \mathrm{m} 2$. This mode of the control mechanism reduces annoying glare when the sun is low in the sky and when the solar irradiation is close to perpendicular to the window. Solar radiation at glancing incidence angles does not turn the window into a reflective state.

O1 - "Office 1" mode corresponds to having the window in "daylight optimization" mode between 7:00 a.m. and 6:00 p.m. and otherwise in "energy optimization" mode.

O2 - "Office 2" mode corresponds to having the window in "daylight optimization" mode during half of the time between 7:00 a.m. and 6:00 p.m. and otherwise in "energy optimization" mode. This is a simplified way of simulating that the office is occupied only during half of the time. 
R1 - "Residential 1" mode corresponds to having the window in "daylight optimization" mode between 6:00 a.m. and 8:00 a.m. and also between 4:00 p.m. and 10:00 p.m. and otherwise in "energy optimization" mode.

R2 - "Residential 2" mode corresponds to having the window in "daylight optimization" mode during half of the time between 6:00 a.m. and 8:00 a.m. and also between 4:00 p.m. and 10:00 p.m. and otherwise in "energy optimization" mode. This is a simplified way of simulating that rooms in the building are only occupied during half of the time.

The different control strategies can easily be modified. Over a year the time resolution of an hour is assumed to be averaged and the simplifications of the strategies is a way to make the results more comprehendible. Switchable windows can then be evaluated and compared to static windows at different locations and in different buildings.

\subsubsection{VIP Energy and eQuest}

VIP Energy is a commercial software simulation tool for whole building simulations and eQuest has been developed by the Lawrence Berkeley National Laboratory and is based on DOE2.2. These software tools are based on dynamic simulation models and thus take the heat storage in the building structure into account. They also take the air flows in the ventilation system and leakage in other building components into account.

Since user precence is assumed to be time dependent and this controls the window state, it is desirable if the software tool can handle time dependent building components. Unfortunately this is not the case for VIP Energy. Instead separate simulations were made for transparent and reflecting states and the energy needs were summarized hour by hour manually. To be able to do this we had to set the indoor temperature to a constant $22^{\circ} \mathrm{C}$ for the whole year. For eQuest the indoor temperature were allowed to vary between $20-26^{\circ} \mathrm{C}$. Other simulation parameters were kept as similar as possible and a list of the most important can be found in table 2 .

Table 2. Common building parameters used in VIP Energy and in eQuest.

\begin{tabular}{cc}
\hline Parameter & Value \\
\hline Climate (from Meteonorm 5.0): & Nagoya \\
Ground reflection & $20 \%$ \\
& \\
Ventilation volume & $14 \mathrm{~m}^{2}$ \\
Floor area & $5.75 \mathrm{~m}^{2}$ \\
& \\
U value, roof & $0.49 \mathrm{~W} / \mathrm{m}^{2} \mathrm{~K}$ \\
U value, south/west wall & $0.33 \mathrm{~W} / \mathrm{m}^{2} \mathrm{~K}$ \\
U value, window & $2.40 / 1.67 \mathrm{~W} / \mathrm{m}^{2} \mathrm{~K}$ \\
\hline
\end{tabular}

\section{Results and Discussion}

\subsection{WinSel}

The results from the WinSel simulations show the importance of the control strategy. For a south facing window for example the energy balance of the window switches from negative to positive with more advanced control strategies for the Nagoya climate as can be seen in figure 1 . The energy balance results are presented per square meter window area. 


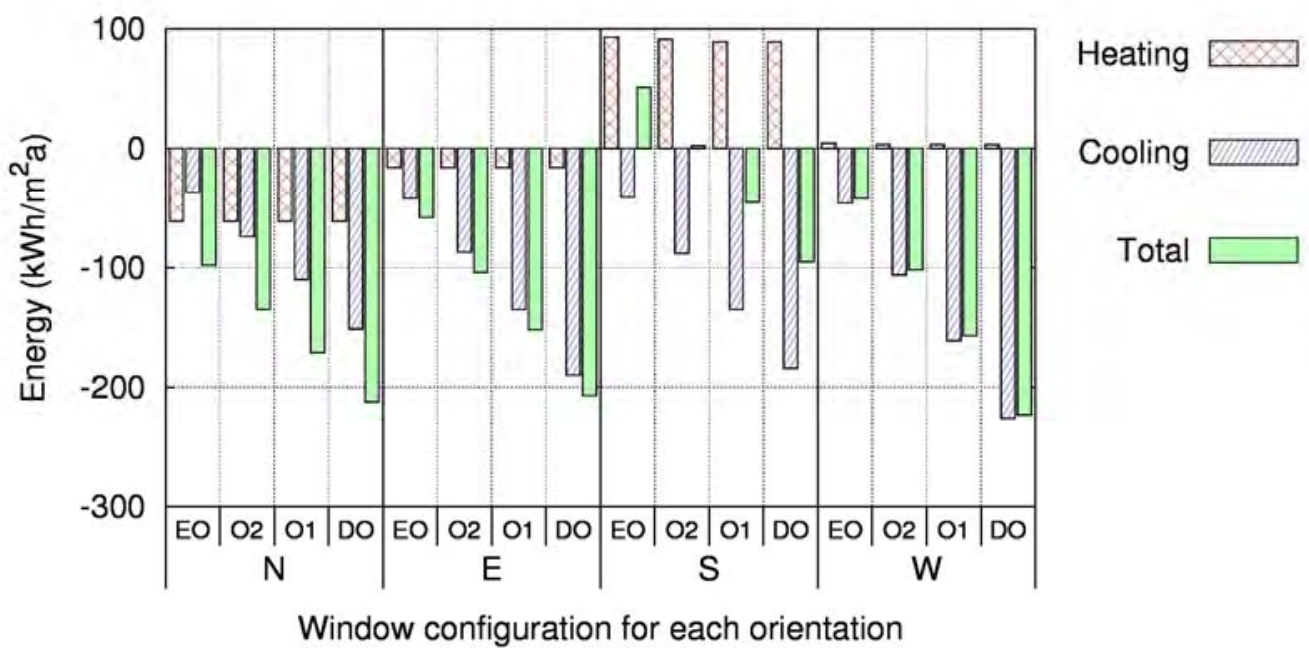

Fig. 1. Simulated energy balance for the test building located in Nagoya using WinSel.

\subsection{VIP Energy}

The whole building simulations made in VIP energy show similar results, found in figure 2, but are presented as energy use per square meter floor area. Comparisons are also made with static windows having g values equal to 30 and $70 \%$ respectively showing that the switchable windows outperform the static windows if using control strategies "O2" (office with presence detectors) or "EO" (switchable window always controlled for a low heating and cooling need.)

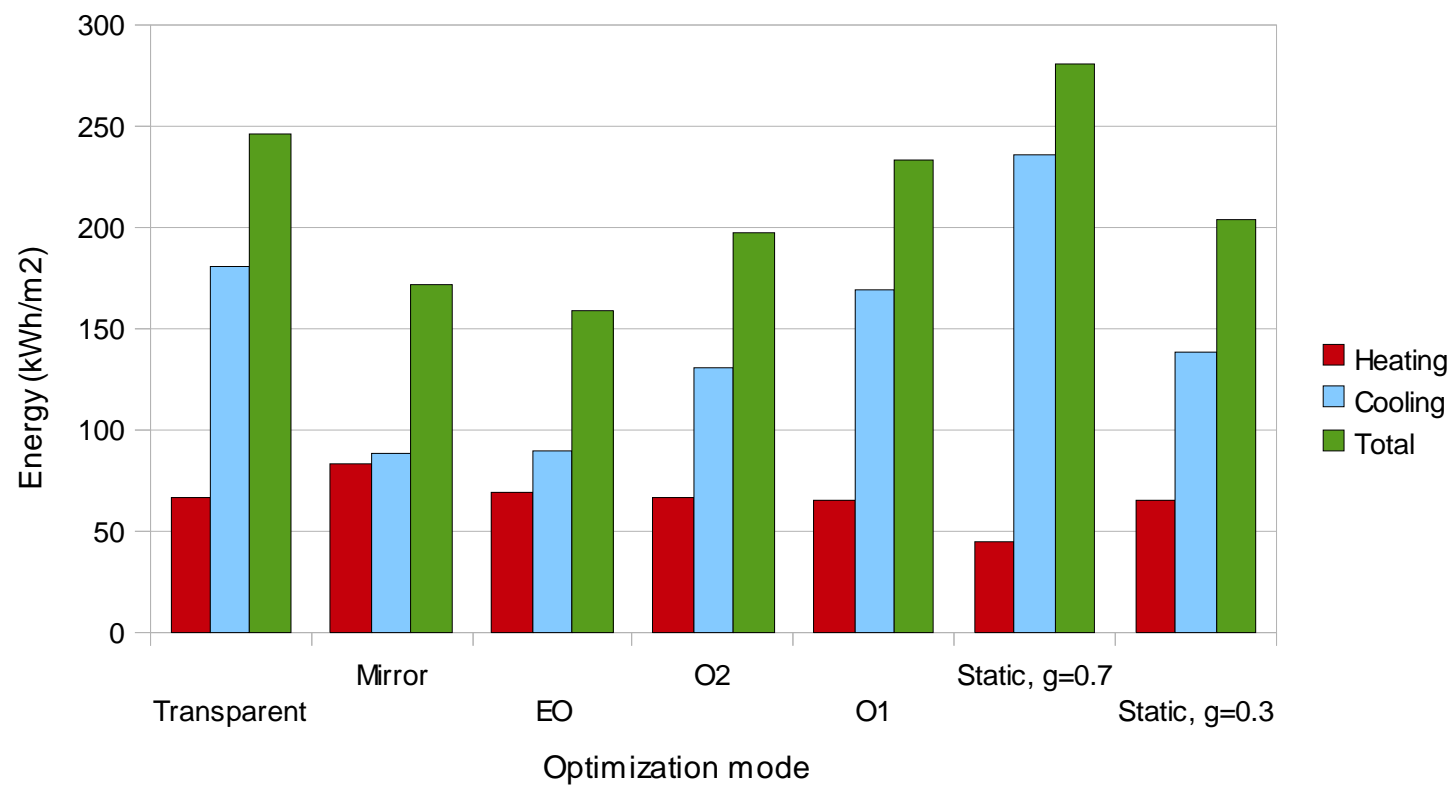

Fig. 2. Simulated energy balance for the test building located in Nagoya using VIP Energy. 


\subsection{Comparisons between three simulation softwares}

Comparisons between simulations made in VIP Energy, eQuest and WinSel show differences of about $20 \%$ for the total energy need. Unfortunately the building models in the simulation softwares cannot be set up in exactly the same way. In VIP Energy there is no built in support for time dependent components. Therefore the indoor temperature had to be set to a constant value at $22^{\circ} \mathrm{C}$ and two separate simulations for transparent and reflective state were made and the results from these simulations were summed up manually. It is reasonable that this leads to a higher than expected energy need, as also can be seen in figure 3 .

The results for WinSel and eQuest are lower but an explanation to the somewhat higher energy need given by the WinSel simulation software might be that the balance temperature is assumed constant over the entire year. One should also remember that this is a static simulation tool and has a very simplified way of handling heat storage within the building.

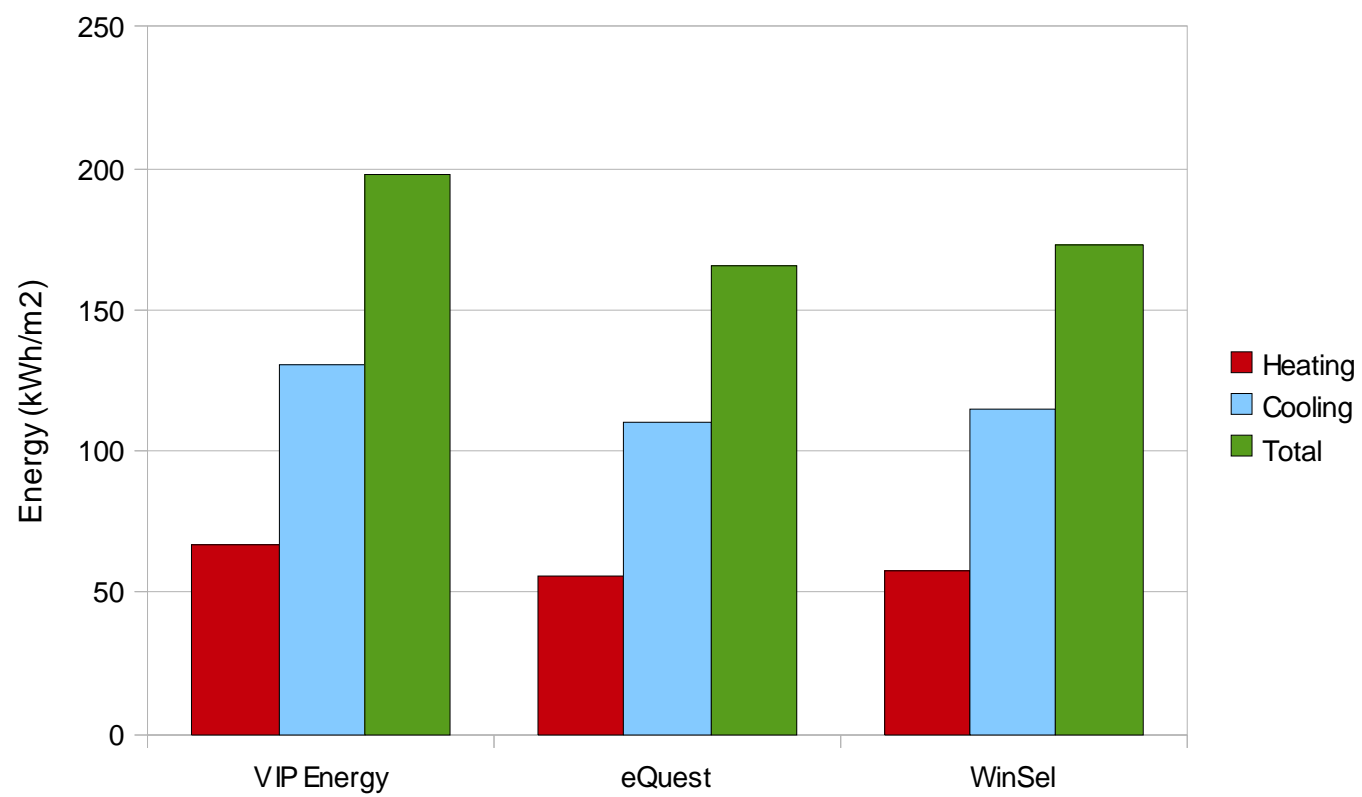

Fig. 3. Simulated energy balances for "O2" optimization mode (office with presence detectors) for three different simulation software tools for the test room located in Nagoya.

\section{Acknowledgement}

This work was supported by the New Energy and Industrial Technology Development Organization program (NEDO).

\section{References}

[1] A. Jonsson, A. Roos, Evaluation of control strategies for different smart window combinations using computer simulations, Solar Energy, 2010, pp. 1 - 9.

[2] A. Jonsson, Optical Characterization and Energy Simulation of Glazing for HighPerformance Windows, Thesis, Uppsala University, 2010.

[3] K. Yoshimura, Y. Yamada, M. Okada, Optical switching of Mg-rich Mg-Ni alloy thin films, Applied Physics Letters 81, 4709, 2002.

[4] VIP Energy software homepage: http://www.strusoft.com/index.php/en/products/vipenergy 
[5] eQuest software homepage: http://doe2.com/equest/

[6] Meteonorm software homepage: http://www.meteonorm.com 